\title{
Significance of hub genes and immune cell infiltration identified by bioinformatics analysis in pelvic organ prolapse
}

\author{
Ying Zhao ${ }^{1}$, Zhijun Xia ${ }^{\text {Corresp., }}{ }^{1}$, Te Lin ${ }^{1}$, Yitong Yin ${ }^{1}$ \\ ${ }^{1}$ Department of Obstetrics and Gynecology, Pelvic Floor Disease Diagnosis and Treatment Center, Shengjing Hospital of China Medical University, \\ Shenyang, China \\ Corresponding Author: Zhijun Xia \\ Email address: xzj18270916865@163.com
}

Objective Pelvic organ prolapse (POP) refers to the decline of pelvic organ position and dysfunction caused by weak pelvic floor support. The aim of the present study was to screen the hub genes and immune cell infiltration related to POP disease. Methods Microarray data of 34 POP tissues in the GSE12852 gene expression dataset were used as research objects. Weighted gene co-expression network analysis (WGCNA) was performed to elucidate the hub module and hub genes related to POP occurrence. Gene function annotation was performed using the DAVID tool. Differential analysis based on the GSE12852 dataset was carried out to explore the expression of the selected hub genes in POP and non-POP tissues, and RT-qPCR was used to validate the results. The differential immune cell infiltration between POP and non-POP tissues was investigated using the CIBERSORT algorithm. Results WGCNA revealed the module that possessed the highest correlation with POP occurrence. Functional annotation indicated that the genes in this module were mainly involved in immunity. ZNF331, THBS1, IFRD1, FLJ20533, CXCR4, GEM, SOD2, and SAT were identified as the hub genes. Differential analysis and RT-qPCR demonstrated that the selected hub genes were overexpressed in POP tissues as compared with non-POP tissues. The CIBERSORT algorithm was employed to evaluate the infiltration of 22 immune cell types in POP tissues and non-POP tissues. We found greater infiltration of activated mast cells and neutrophils in POP tissues than non-POP tissues, while the infiltration of resting mast cells was lower in POP tissues. Moreover, we investigated the relationship between the type of immune cell infiltration and hub genes by Pearson correlation analysis. The results indicate that activated mast cells and neutrophils had a positive correlation with the hub genes, while resting mast cells had a negative correlation with the hub genes. Conclusions Our research identified 8 hub genes and the infiltration of 3 types of immune cells related to POP occurrence. These hub genes may participate in the pathogenesis of POP through the immune system, giving them a certain diagnostic and therapeutic value. 
1 Significance of hub genes and immune cell infiltration identified by bioinformatics

2 analysis in pelvic organ prolapse

3 Ying Zhao ${ }^{1}$, Zhijun Xia*1, Te Lin ${ }^{1}$, Yitong Yin ${ }^{1}$

$4{ }^{1}$ Department of Obstetrics and Gynecology, Pelvic Floor Disease Diagnosis and Treatment Center,

5 Shengjing Hospital of China Medical University, Shenyang, China, 110004.

6 *Correspondence:

7 Corresponding Author: Zhijun Xia, Department of Obstetrics and Gynecology, Shengjing Hospital

8 of China Medical University, Shenyang, China, 110004

9 Email: xzj18270916865@163.com,xiazj@sj-hospital.org

10 Acknowledgments

11 We acknowledge the authors who provided information for the GEO public database.

12 Funding statement: This article was funded by Shengjing Hospital of China Medical University.

13 Abstract

14 Objective Pelvic organ prolapse (POP) refers to the decline of pelvic organ position and 15 dysfunction caused by weak pelvic floor support. The aim of the present study was to screen the 16 hub genes and immune cell infiltration related to POP disease.

17 Methods Microarray data of 34 POP tissues in the GSE12852 gene expression dataset were used as research objects. Weighted gene co-expression network analysis (WGCNA) was performed to elucidate the hub module and hub genes related to POP occurrence. Gene function annotation was performed using the DAVID tool. Differential analysis based on the GSE12852 dataset was carried out to explore the expression of the selected hub genes in POP and non-POP tissues, and RT-qPCR was used to validate the results. The differential immune cell infiltration between POP and nonPOP tissues was investigated using the CIBERSORT algorithm.

24 Results WGCNA revealed the module that possessed the highest correlation with POP occurrence.

25 Functional annotation indicated that the genes in this module were mainly involved in immunity. 26 ZNF331, THBS1, IFRD1, FLJ20533, CXCR4, GEM, SOD2, and SAT were identified as the hub 
27 genes. Differential analysis and RT-qPCR demonstrated that the selected hub genes were overexpressed in POP tissues as compared with non-POP tissues. The CIBERSORT algorithm was employed to evaluate the infiltration of 22 immune cell types in POP tissues and non-POP tissues. We found greater infiltration of activated mast cells and neutrophils in POP tissues than non-POP tissues, while the infiltration of resting mast cells was lower in POP tissues. Moreover, we investigated the relationship between the type of immune cell infiltration and hub genes by Pearson correlation analysis. The results indicate that activated mast cells and neutrophils had a positive correlation with the hub genes, while resting mast cells had a negative correlation with the hub genes.

Conclusions Our research identified 8 hub genes and the infiltration of 3 types of immune cells related to POP occurrence. These hub genes may participate in the pathogenesis of POP through the immune system, giving them a certain diagnostic and therapeutic value.

Keywords: Pelvic organ prolapse, WGCNA, CIBERSORT, immune cell infiltration, GEO database

\section{Introduction}

Pelvic organ prolapse (POP) is caused by dysfunction of the pelvic floor supporting structures and affects the quality of life of many women (Nygaard et al. 2014). The prevalence of POP is expected to reach $46 \%$ by 2050 (Wu et al. 2009). Currently, only approximately $20 \%$ of women have POP surgery during their lifetime (Haya et al. 2018); however, the reoperation rate is high (Friedman et al. 2018), which causes a huge economic burden. POP is a complex, multifaceted disease resulting from the interaction between environmental and genetic factors. Pregnancy, vaginal pull-up, time of delivery, age, and obesity have been identified as risk factors (Hendrix et al. 2002); nevertheless, the molecular mechanism of POP remains unclear, and there is a lack of suitable prevention and treatment measures in clinical practice. Therefore, it is imperative to 
52 explore the molecular mechanism of POP occurrence for the benefit for both women and society.

53 Owing to the development of gene chips and new generation sequencing technology,

54 bioinformatics analysis plays an increasingly important role in biomedical research. Weighted

55 gene co-expression network analysis (WGCNA) can identify gene module characteristics and hub

56 genes to connect the gene modules and sample characteristics (Wang et al. 2020b). The

57 CIBERSORT algorithm can be employed to evaluate immune cell infiltration in tissues based on

58 gene expression datasets. Recently, abundant researches have used this algorithm to explore the

59 function of immune cells in diseases(Lin et al. 2020), breast ductal and lobular carcinoma (Zhang

60 et al. 2019), osteoarthritis (Cai et al. 2020), and high-grade serous ovarian cancer (Liu et al. 2020).

61 Our study found hub genes and immune cells highly related to POP occurrence by analyzing POP

62 expression spectrum data in public databases using WGCNA and the CIBERSORT algorithm,

63 providing novel ideas and methods for the treatment of POP. Finally, the differential immune cells

64 was investigated between POP and non-POP tissues.

\section{Materials and Methods}

66

67

\section{Obtaining the training and validation POP datasets}

The workflow diagram is presented in Figure 1. The training dataset was obtained from the Gene Expression Omnibus database (GEO, https://www. ncbi.nlm. nih. gov/) in NCBI based on the keywords "pelvic organ prolapse" and "gene expression profiles" and "Homo sapiens" (Brizzolara et al. 2009). In addition, we selected a dataset with a sample size greater than 30 , which is the minimum sample size required to construct a WGCNA network (POP $>15$, non-POP $>15$ ); the GSE12852 dataset, containing 16 POP and 18 non-POP patients, was the only dataset to meet this condition. The gene expression profiles and corresponding clinical information including age, menopausal status, race, and prolapse stage were acquired from the GSE12852 dataset. Subsequently, log-scale robust multi-array analysis was used to perform background correction and normalization of the datasets. A total of 12 POP patients enrolled at the Shengjing Hospital of 
77 China Medical University from 2017 to 2018 were selected as the validation dataset. The Ethics

78 Committee of Shengjing Hospital of China Medical University approved the study protocol in 79 accordance with the guidelines of the Declaration of Helsinki (No. 2018Ps68K), and our study was 80 exempt from consent. Prolapse tissues were selected as the experimental group and non-prolapse 81 tissues were selected as the control group. All patients were married, without estrogen-related 82 disorders, and had received no hormone treatment within the previous 3 months. Basic information 83 of the patients in the validation dataset is presented in Supplementary Table 1.

\section{Co-expression network construction based on the training dataset}

85 We used the "WGCNA" package of the R software to establish the network based on the 34 86 samples in the training dataset according to a previously described method (Chen et al. 2018b; 87 Zhou et al. 2018). Firstly, POP and normal samples were analyzed using the "flash cluster" 88 package of the R software to remove outlier samples. The correlation matrix of gene co-expression consists of the correlation coefficient between two genes. The average linkage matrix and Pearson correlation method were performed to construct the correlation matrix between genes.

91 Subsequently, the formula amn $=|\mathrm{cmn}| \beta$ was used to convert the correlation matrix to the 92 adjacency matrix (amn represents the correlation coefficient between gene $\mathrm{m}$ and gene $\mathrm{n}$; $\mathrm{cmn}$ 93 represents the connection coefficient between gene $\mathrm{m}$ and gene $\mathrm{n}$; and $\beta$ is a soft threshold that 94 can strengthen strong links between genes and weaken weak links). Finally, we converted the 95 adjacency matrix to the topological overlap matrix (TOM) based on an appropriate soft threshold, 96 and placed the similar genes into the same module.

\section{Identification of hub gene modules and genes}

98 Similar genes were placed into the same module based on the WGCNA co-expression network.

99 The main purpose of our research was to combine clinical information (POP or non-POP samples) 100 with gene modules to analyze gene significance (GS) and modular membership (MM). MM means 101 the correlation between gene expression profile level and module eigengenes (ME). ME are 102 considered the correlation between modules and clinical information. GS represents the degree of 
103 correlation between gene expression profiles and clinical information. The average value of all

104 gene GS in the module represents the module significance (MS). We defined the correlation

105 between gene and disease as GS, and obtained the correlation between this module and disease as

106 MS. The hub genes characterized by a high MM and a high GS are described as having the closest

107 relationship with disease. In our research, the module with the highest MS was selected as the hub

108 module. The genes in the hub module with $|\mathrm{MM}|>0.8$ and $|\mathrm{GS}|>0.47$ were considered hub

109 genes.

\section{Functional enrichment analysis}

111 We used the online GO enrichment analysis and KEGG pathway analysis tools on the DAVID

112 website to annotate the genes in the module identified by WGCNA, and attempted to elucidate the

113 enrichment pathway and functions of the target genes. The Human Genome U133 Plus 2 Array

114 was used as the background data (The FDR $<0.01)$.

\section{Immune cell infiltration in POP tissues}

116 CIBERSORT is an algorithm to characterize the proportion of 22 immune cells (Supplementary

117 Table 2) in tissues using 547 barcode gene expression values. The CIBERSORT algorithm was

118 employed to elucidate the proportion of 22 immune cells in POP tissues. The samples with $p$ value

$119<0.05$ were significant (Chen et al. 2018a). Pearson correlation analysis was implemented to

120 obtain the related coefficient between the 22 immune cells. Then, we investigated the differential

121 immune cell infiltration between POP and non-POP tissues. Finally, we calculated the related

122 coefficient between infiltration of different immune cells and hub genes by Pearson correlation 123 analysis.

\section{RNA extraction and quantitative real-time PCR based on the validation dataset}

125 TRIzol® $(1 \mathrm{~mL})$ was used to isolate total RNA from POP tissues $(200 \mathrm{mg})$ in the validation dataset, 126 and reverse transcriptase from the avian myeloblastoma virus and random primers were used to 127 create complementary DNA (cDNA) according to the instructions from TAKARA. SYBR Premix 128 Ex Taq II (Takara) was used to amplify the cDNA. According to the samples from three 
129 independent experiments, the $2^{-\Delta \Delta \mathrm{CT}}$ value was used to analyze the data. Primers for the genes are

130 displayed in Supplementary Table 3.

131 Statistical analysis

132 The GraphPad Prism 7.0 and R 3.6.1 software were used for statistical analysis and image

133 generation. A $t$-test was employed to analyze the differences between two groups. The $p<0.05$

134 was statistically significant.

1353 Results

\section{Construction of WGCNA and identification of hub modules}

137 The "flash cluster" software package in R was used to cluster the 34 samples in the GSE12852

138 dataset to remove the outlier samples; 4 outlier samples were removed (Figure 2). There were few

139 differences among the remaining samples, which is conducive to the accuracy of the results. The

140 correlation matrix between genes was calculated according to the average linkage matrix and

141 Pearson correlation method. The formula amn $=|\mathrm{cmn}| \beta(\beta=7)$ was used to transform the

142 correlation matrix into the adjacency matrix. In order to better construct a scale-free network

143 distribution, the "picks of threshold" function of "WGCNA" package calculated the value of

144 parameter $\beta$. In POP and non-POP samples, 1-20 thresholds were selected and the correlation

145 coefficient, mean connectivity, and average correlation degree between $\log (\mathrm{k})$ and $\log (\mathrm{P}(\mathrm{k}))$ were

146 calculated for each threshold. At this time, the average network connectivity corresponding to the

147 threshold is close to zero, indicating that the network connectivity is very low, which is similar to

148 the scale-free network (Figure 3). According to the corresponding steps of WGCNA, the gene

149 network was built after the hierarchical clustering tree. In this experiment, the dynamic pruning

150 tree method merged with similar genes into one gene module. The minimum number of genes in

151 a module was 30, and 11 modules were obtained (Figure 4A). According to the thermogram of

152 correlation between modules and clinical information, the highest correlation existed in the blue

153 module and sample type, with a correlation coefficient of 0.47 (Figure 4B, $p=0.009$ ). Therefore,

154 the blue module was considered the most study-worthy. Protein-protein interactions (PPIs) are 
155 widely involved in the process of vital movement. http://omnipathdb.org/ was used to construct

156 the PPI network for genes in the blue module (Figure 5)(Türei et al. 2016).

157 Functional enrichment analysis

158 To further study the biological function of the genes in the blue module, GO and KEGG

159 enrichment analyses were used. GO enrichment analysis showed that these genes mainly

160 participated in the immune regulation (Figure 6A, Supplementary Table 4). KEGG enrichment

161 analysis identified that these genes participated in the regulation of immune-related pathways, such

162 as the IL-17 and TNF signaling pathways (Figure 6B, Supplementary Table 5).

\section{Identification of the hub genes}

164 According to the screening criteria $|\mathrm{MM}|>0.8$ and $|\mathrm{GS}|>0.47,8$ genes $($ ZNF331, THBS1,

$165 T M E M 70, C X C R 4, G E M, S O D 2$, and $S A T$ ) were identified in the blue module as hub genes (Figure

166 7A). We found that the hub genes were highly expressed in POP tissues as compared with non-

167 POP tissues by differential analysis based on the GSE12852 dataset (Figure 7B). From the

168 heatmap, it can be seen that hub genes are overexpressed in POP tissues (Figure 7C). In addition,

169 the correlation coefficient between the hub genes was calculated, which showed that there was a

170 strong co-expression relationship between these genes (Figure 7D).

\section{Validation of the hub genes}

172 To verify the accuracy of the prediction results, RT-qPCR was used to detect the expression of the

173 hub genes in 12 pairs of POP and non-POP tissues. The results show that the hub genes were

174 overexpressed in POP tissues, which is consistent with the prediction results (Figure 8A-H).

\section{Immune cell infiltration analysis}

176 The CIBERSORT algorithm was employed to select samples with a output $p<0.05$. 12 samples

177 including 4 non-POP and 8 POP tissues were obtained. A bar plot was generated to show the

178 proportion of 22 immune cells in the 12 samples (Figure 9A). We found that macrophages account

179 for the largest proportion among the immune cells in the samples. Figure 9B indicates that M1

180 macrophages had the strongest positive correlation with resting mast cells (correlation coefficient, 
181 0.58), whereas resting mast cells had the strongest negative correlation with activated mast cells

182 (correlation coefficient, 0.83). We found a higher infiltration of activated mast cells and

183 neutrophils in POP tissues than in non-POP tissues, while the infiltration of resting mast cells was

184 lower in POP tissues (Figure 10, $<<0.05$ ). Finally, Pearson correlation analysis was used to 185 calculate the related coefficient between the infiltration of different immune cells and hub genes.

186 The results reveal that activated mast cells and neutrophils had a positive correlation with the hub

187 genes, while resting mast cells had a negative correlation with the hub genes (Table 1).

\section{Discussion}

189 Pelvic organ prolapse (POP) is caused by weakening of the supporting structures of the pelvic

190 floor, resulting in the position of pelvic floor organs moving downward and the function becoming 191 abnormal. POP seriously affects the quality of life of middle-aged and elderly women. In recent 192 years, with the rapid development of gene sequencing and bioinformatics technologies, further 193 analysis and utilization of sequencing data have become possible. To explore the molecular 194 mechanism related to the development of POP, 8 hub genes (ZNF331, THBS1, IFRD1, FLJ20533, 195 CXCR4, GEM, SOD2, and SAT) related to POP occurrence were screened out based on WGCNA 196 and their biological functions were explored.

197 Thrombospondins (THBSs) are a group of glycoproteins that bind to collagen and tissue,

198 participating in the interaction between cells and the extracellular matrix during the process of 199 tissue development and repair. THBS1 is the first member of the THBS gene family, which plays 200 a significant role in many biological processes related to the occurrence and progression of 201 cardiovascular diseases, such as angiogenesis, inflammation, and tissue remodeling (Zhao et al. 202 2018). In addition, THBS1 can also affect tumor cell adhesion, invasion, migration, proliferation, 203 apoptosis, and immune evasion (Huang et al. 2017). The overexpression of THBS1 in POP tissues 204 as compared with non-POP tissues has been predicted and verified (Brizzolara et al. 2009), which 205 is consistent with the results of our secondary analysis. ZNF331 is located on chromosome 19q13, 
206 a recently cloned gene encoding a zinc-finger protein involved in thyroid tumorigenesis (Babinger 207 et al. 2007). ZNF331, as a tumor suppressor gene, has also been reported to have low expression 208 in colorectal cancer (Wang et al. 2017), esophageal cancer (Jiang et al. 2015), gastric cancer (Yu 209 et al. 2013), and liver cancer (Wang et al. 2013), and its low expression is related to 210 hypermethylation of its promoter region. However, the research on ZNF331 in other diseases was 211 rare. Interferon-related developmental regulator 1 (IFRDI) is located on chromosome 7q22-q31

212 and acts a significant role in the development and differentiation of embryonic muscle cells (Kraus 213 et al. 2001; Lincoln et al. 2004). IFRDI has been proved to be a modified gene of cystic fibrosis 214 lung disease, which can regulate the effector function of neutrophil(Gu et al. 2009a). 215 Transmembrane protein 70 (TMEM70), also named FLJ20533, is a mitochondrial membrane 216 protein that acts a role in the biosynthesis of mitochondrial ATPase (Hejzlarova et al. 2011). At 217 present, research related to TMEM70 mainly focuses on cardiomyopathy and pulmonary 218 hypertension. CXCR4, also known as CD184, is a highly conserved receptor for the chemokine 219 CXCL12 (Kashyap et al. 2017). CXCR4 belongs to the G protein-coupled receptor superfamily 220 and is expressed in a cortical protein-dependent manner on the cell surface (Teicher \& Fricker 221 2010). CXCR4 has been reported to be overexpressed in a variety of tumor cells and is involved in tumor proliferation, invasion, metastasis, and worse prognosis (Ottaiano et al. 2020; Wang et al. 2020a; Zhu et al. 2020). GTP-binding protein overexpressed in skeletal muscle (GEM) is a 224 GTPase originally identified in mitogen-stimulated $\mathrm{T}$ lymphocytes and v-Abl-transformed pre-B 225 cells and is highly expressed in the spleen, thymus, and kidneys (Cohen et al. 1994; Huang et al. 226 2014). Mitochondrial superoxide dismutase (SOD2) is an antioxidant enzyme that reduces the 227 damage caused by oxidative stress to protect mitochondria (Koltai et al. 2018). 228 Spermidine/spermine N1-acetyltransferase (SAT) is the rate-limiting enzyme in polyamine 229 catabolism and functions through the acetylation of spermidine and spermidine to affect cell 230 growth, proliferation, and apoptosis (Pegg 2008; Pegg 2016). Despite a detailed literature review, 231 we found no reports of the involvement ZNF331, IFRD1, FLJ20533, CXCR4, GEM, SOD2, or 
232 SAT in POP. To explore the expression of the selected hub genes in POP and non-POP tissues, 233 differential analysis of the GSE12852 dataset was performed by RT-qPCR. The results indicate 234 that the selected hub genes were overexpressed in POP tissues as compared with non-POP tissues, 235 suggesting that the selected hub genes may be related to POP occurrence.

236 GO and KEGG functional enrichment analyses were performed to study the function of the 237 hub genes using the "R" software. GO functional annotation showed that the hub genes were 238 mainly participated in the immune response. KEGG enrichment analysis revealed that the hub 239 genes participated in the regulation of immune-related pathways such as the IL-17 signaling 240 pathway. IL-17 is a member of the inflammatory cytokine family and is mainly produced by Th17

241 cells. IL-17 signaling has been reported to be associated with immunopathology and autoimmune 242 diseases (Amatya et al. 2017). Previous research has confirmed that the extracellular matrix 243 components in connective tissue can control the physical strength of the pelvic floor (Dietz et al. 244 2002). The immune system can maintain the homeostasis of POP tissues by adjusting extracellular 245 matrix components (Yu et al. 2010); thus, it is reasonable to suggest that regulation of the immune 246 system is closely related to the occurrence of POP. At present, there is no direct evidence indicating 247 that IL-17 is related to POP occurrence; however, we have reason to believe that the IL-17 248 signaling pathway plays a significant role in the development of POP given its role in immune 249 regulation. In view of the close relationship between hub genes and immunity, we investigated 250 immune cell infiltration in POP and non-POP tissues using the CIBERSORT algorithm. We found 251 a higher infiltration of activated mast cells and neutrophils in POP tissues than in non-POP tissues, 252 while infiltration of resting mast cells was lower in POP tissues. Activated mast cells and 253 neutrophils had a positive correlation with the hub genes, while resting mast cells had a negative 254 correlation. According to the results, it is reasonable to suggest that the hub genes may be related 255 to the development of POP by regulating the levels of activated/resting mast cells and neutrophils. 256 Mast cells are important antigen-presenting cells that can release histamine and cytokines through 257 degranulation and act a significant role in the occurrence and development of various inflammatory 
258 diseases (Grabauskas et al. 2020; Novruzov 2008; Sajay-Asbaghi et al. 2020). Very recently,

259 THBS1 was demonstrated to promote the inflammatory response of mast cells in CIU (chronic 260 idiopathic urticaria) and the permeability of HDMECs (human dermal microvascular endothelial 261 cells) by regulating the TGF- $\beta /$ SMAD pathway, the effects of which can be inhibited by miR-194 262 (Qu et al. 2020). Several studies have shown that CXCR4 can promote mast cell chemotaxis to 263 inflammatory sites (Limón-Flores et al. 2009; Lv et al. 2019; Patadia et al. 2010), and IFRD1 may 264 involve in neutrophilic inflammation in cystic fibrosis (Blanchard et al. 2011; Gu et al. 2009b; 265 Hector et al. 2013). According to the current literature, there is no direct evidence to prove the 266 accuracy of our prediction results; however, the relationship between the hub genes and infiltration 267 of different immune cells suggests they are correct.

268 Some limitations of this research need to be discussed. Firstly, the research remained at the 269 prediction stage and there is insufficient experimental evidence to verify our prediction results. In 270 the future, verification in vitro and in vivo should strengthen our observations. Secondly, the 271 sample size for this study was not large enough due to the limited datasets in the database, which 272 may have caused some bias. Thirdly, the samples of our research were coming from the 273 ligamentum. The ligamentum are full of fibroblasts and it is not sure that they capture many white 274 blood cells. We could not guarantee the accuracy of the CIBERSORT algorithms completely due 275 to the blood specificity of the algorithms.

\section{Conclusions}

277 Our research identified 8 hub genes and 3 immune cell types that may be related to POP 278 occurrence. These hub genes may participate in the pathogenesis of POP by regulating the immune 279 environment, giving them certain diagnostic and therapeutic value in POP.

\section{Acknowledgments}

281 We thank the authors who provided the GEO public datasets.

\section{Conflict of Interest Statement}

283 The authors declare no competing interests. 
287 FIGURE 1. Workflow diagram. Data collection, analysis and validation.

FIGURE 2. Clustering dendrogram of 34 POP samples in GSE12852. POP samples were assigned as 1 ; non-POP samples were assigned as 0 . Pre-menopause status samples were assigned as 0 ; menopause status samples were assigned as 1 . Red color intensity is proportional to POP samples, pre-menopause status, and higher age.

293

FIGURE 3. Identification of the soft threshold in the scale-free network.

294

(A) Different soft-threshold and corresponding scale free topology model(B) Different softthresholding powers and corresponding mean connectivity. (C) The distribution of the connectedness by histogram. (D) Inspection of the scale free topology.

\section{FIGURE 4. Identification of the hub module.}

(A) Dendrogram showed the genes with similar function classed into the same module according to the dissimilarity measure. (B) Heatmap revealed the correlation between modular eigengenes (ME) and clinical information of POP.

FIGURE 5. PPI network analysis.

302 PPI network analysis of the genes in the blue module based on the OmniPath online database. Nodes represent the hub genes in the blue module identified by WGCNA. Lines represent interactions between hub genes.

\section{FIGURE 6. GO functional and KEGG pathway enrichment analysis.}

(A) Biological process (BP), (B)cellular component (CC) and (C) molecular function (MF) of GO analysis using the "org.Hs.eg.db" package in the R software. 
310 (A) The relationship between gene MM and GS. (B) Differential expression of the hub genes. (C)

311 Heatmap of the hub genes in GSE12852. (D) Correlation analysis of the hub genes in GSE12852.

312 FIGURE 8. Hub gene validation.

313 (A-H) RT-qPCR detection of the expression of hub genes in 12 pairs of POP and non-POP tissues.

314 FIGURE 9. The landscape of immune cell infiltration in GSE12852 (CIBERSORT $p$ value $<$ 315 0.05).

316 (A) Proportion of the 22 immune cell types in GSE12852. (B) Correlation matrix between the 22 317 immune cell types.

318 FIGURE 10. The differential immune cell infiltration between POP and non-POP tissues.

319 (A) Resting mast cells; (B) activated mast cells; (C) neutrophils. Group 1: non-POP; Group 2: 320 POP.

321 TABLE 1 . The correlation between hub genes and immune cell infiltration

322 SUPPLEMENTARY TABLE 1. Basic information of the patients in the validation dataset

323 SUPPLEMENTARY TABLE 2 . The 22 immune cells

324 SUPPLEMENTARY TABLE 3.The primers for the hub genes

325 SUPPLEMENTARY TABLE 4. GO functional enrichment analysis

326 SUPPLEMENTARY TABLE 5. KEGG pathway enrichment analysis

327

328

\section{Reference}

329

330

331

332

333

334

335

336

337

338

Amatya N, Garg AV, and Gaffen SL. 2017. IL-17 Signaling: The Yin and the Yang. Trends Immunol 38:310-322. 10.1016/j. it. 2017.01.006

Babinger P, Volk1 R, Cakstina I, Maftei A, and Schmitt R. 2007. Maintenance DNA methyltransferase (Met1) and silencing of $\mathrm{CpG}^{-}$-methylated foreign DNA in Volvox carteri. Plant Mol Biol 63:325-336. 10.1007/s11103-006-9091-1

Blanchard E, Marie S, Riffault L, Bonora M, Tabary 0, Clement A, and Jacquot J. 2011. Reduced expression of Tis7/IFRD1 protein in murine and human cystic fibrosis airway epithelial cell models homozygous for the F508de1-CFTR mutation. Biochemical and biophysical research communications 411:471- 
339

340

341

342

343

344

345

346

347

348

349

350

351

352

353

354

355

356

357

358

359

360

361

362

363

364

365

366

367

368

369

370

371

372

373

374

375

376

377

476. 10. 1016/ j. bbrc. 2011. 06. 104

Brizzolara SS, Killeen J, and Urschitz J. 2009. Gene expression profile in pelvic organ prolapse. Mol Hum Reprod 15:59-67. 10.1093/molehr/gan074

Cai W, Li H, Zhang Y, and Han G. 2020. Identification of key biomarkers and immune infiltration in the synovial tissue of osteoarthritis by bioinformatics analysis. PeerJ 8:e8390-e8390. 10.7717/peerj. 8390

Chen B, Khodadoust MS, Liu CL, Newman AM, and Alizadeh AA. 2018a. Profiling Tumor Infiltrating Immune Cells with CIBERSORT. Methods Mol Biol 1711:243259. 10. 1007/978-1-4939-7493-1_12

Chen Y, Bi F, An Y, and Yang Q. 2018b. Coexpression network analysis identified Kruppe1-1ike factor 6 (KLF6) association with chemosensitivity in ovarian cancer. J Cell Biochem. 10.1002/jcb. 27567

Cohen L, Mohr R, Chen YY, Huang M, Kato R, Dorin D, Tamanoi F, Goga A, Afar D, Rosenberg N. 1994. Transcriptional activation of a ras-like gene (kir) by oncogenic tyrosine kinases. Proc Natl Acad Sci U S A 91:12448-12452. 10. 1073/pnas. 91.26. 12448

Dietz HP, Jarvis SK, and Vancaillie TG. 2002. The assessment of levator muscle strength: a validation of three ultrasound techniques. Int Urogynecol J Pelvic Floor Dysfunct 13:156-159; discussion 159. 10.1007/s192-002-83465

Friedman T, Eslick GD, and Dietz HP. 2018. Risk factors for prolapse recurrence: systematic review and meta-analysis. Int Urogynecol $J$ 29:13-21. 10. $1007 / \mathrm{s} 00192-017-3475-4$

Grabauskas G, Wu X, Gao J, Li J-Y, Turgeon DK, and Owyang C. 2020. Prostaglandin E2, Produced by Mast Cells in Colon Tissues from Patients with Irritable Bowel Syndrome, Contributes to Visceral Hypersensitivity in Mice. Gastroenterology:S0016-5085 (0020) 30232-30238.

10. 1053/j. gastro. 2020.02. 022

Gu Y, Harley IT, Henderson LB, Aronow BJ, Vietor I, Huber LA, Harley JB, Kilpatrick JR, Langefeld CD, Williams AH, Jegga AG, Chen J, Wills-Karp M, Arshad SH, Ewart SL, Thio CL, Flick LM, Filippi MD, Grimes HL, Drumm ML, Cutting GR, Knowles MR, and Karp CL. 2009a. Identification of IFRD1 as a modifier gene for cystic fibrosis lung disease. Nature 458:1039-1042. 10. 1038/nature07811

Gu Y, Harley ITW, Henderson LB, Aronow BJ, Vietor I, Huber LA, Harley JB, Kilpatrick JR, Langefeld CD, Williams AH, Jegga AG, Chen J, Wil1s-Karp M, Arshad SH, Ewart SL, Thio CL, Flick LM, Filippi M-D, Grimes HL, Drumm ML, Cutting GR, Knowles MR, and Karp CL. 2009b. Identification of IFRD1 as a modifier gene for cystic fibrosis lung disease. Nature 458:1039-1042. 
378

379

380

381

382

383

384

385

386

387

388

389

390

391

392

393

394

395

396

397

398

399

400

401

402

403

404

405

406

407

408

409

410

411

412

413

414

415

416

10. 1038/nature07811

Haya N, Feiner B, Baessler K, Christmann-Schmid C, and Maher C. 2018. Perioperative interventions in pelvic organ prolapse surgery. Cochrane Database Syst Rev 8:Cd013105. 10.1002/14651858. Cd013105

Hector A, Kormann M, Kammermeier J, Burdi S, Marcos V, Rieber N, Mays L, I1lig T, Klopp N, Falkenstein F, Kappler M, Riethmueller J, Graepler-Mainka U, Stern M, Eickmeier 0, Serve F, Zielen S, Döring G, Griese M, and Hart1 D. 2013. Expression and regulation of interferon-related development regulator-1 in cystic fibrosis neutrophils. American journal of respiratory cell and molecular biology 48:71-77. 10.1165/rcmb. 2012-00610C

Hejzlarova K, Tesarova M, Vrbacka-Cizkova A, Vrbacky M, Hartmannova H, Kaplanova V, Noskova L, Kratochvilova H, Buzkova J, Havlickova V, Zeman J, Kmoch S, and Houstek J. 2011. Expression and processing of the TMEM70 protein. Biochim Biophys Acta 1807:144-149. 10.1016/j. bbabio. 2010.10. 005

Hendrix SL, Clark A, Nygaard I, Aragaki A, Barnabei V, and McTiernan A. 2002. Pelvic organ prolapse in the Women's Health Initiative: gravity and gravidity. Am J Obstet Gynecol 186:1160-1166. 10.1067/mob. 2002. 123819

Huang T, Sun L, Yuan X, and Qiu H. 2017. Thrombospondin-1 is a multifaceted player in tumor progression. Oncotarget 8:84546-84558. 10. 18632 /oncotarget. 19165

Huang X, Cong X, Yang D, Ji L, Liu Y, Cui X, Cai J, He S, Zhu C, Ni R, and Zhang Y. 2014. Identification of Gem as a new candidate prognostic marker in hepatocellular carcinoma. Pathology, research and practice 210:719-725. 10. 1016/j. prp. 2014. 07. 001

Jiang S, Linghu E, Zhan Q, Han W, and Guo M. 2015. Methylation of ZNF331 Promotes Ce11 Invasion and Migration in Human Esophageal Cancer. Curr Protein Pept Sci 16:322-328. 10.2174/138920371604150429155255

Kashyap MK, Amaya-Chanaga CI, Kumar D, Simmons B, Huser N, Gu Y, Hallin M, Lindquist K, Yafawi R, Choi MY, Amine A-A, Rassenti LZ, Zhang C, Liu SH, Smeal T, Fantin VR, Kipps TJ, Pernasetti F, and Castro JE. 2017. Targeting the CXCR4 pathway using a novel anti-CXCR4 IgG1 antibody (PF06747143) in chronic lymphocytic leukemia. J Hematol Oncol 10:112-112. 10. $1186 / \mathrm{s} 13045-017-0435-\mathrm{x}$

Koltai E, Bori Z, Osvath P, Ihasz F, Peter S, Toth G, Degens H, Rittweger J, Boldogh I, and Radak Z. 2018. Master athletes have higher miR-7, SIRT3 and SOD2 expression in skeletal muscle than age-matched sedentary controls. Redox biology 19:46-51. 10.1016/j. redox. 2018. 07.022

Kraus F, Haenig B, and Kispert A. 2001. Cloning and expression analysis of the mouse T-box gene tbx20. Mech Dev 100:87-91. 10.1016/s0925-4773(00) 00499- 
$417 \quad 8$

418 Limón-Flores AY, Chacón-Salinas R, Ramos G, and U11rich SE. 2009. Mast cel1s 419 mediate the immune suppression induced by dermal exposure to JP-8 jet

420

421

422

423

424

425

426

427

428

429

430

431

432

433

434

435

436

437

438

439

440

441

442

443

444

445

446

447

448

449

450

451

452

453

454

455 fuel. Toxicological sciences : an official journal of the Society of Toxicology 112:144-152. 10. 1093/toxsci/kfp181

Lin J, Yu M, Xu X, Wang Y, Xing H, An J, Yang J, Tang C, Sun D, and Zhu Y. 2020. Identification of biomarkers related to $\mathrm{CD} 8(+) \mathrm{T}$ cell infiltration with gene co-expression network in clear cell renal cell carcinoma. Aging (Albany NY) 12:10.18632/aging. 102841. 10.18632/aging. 102841

Lincoln J, Alfieri CM, and Yutzey KE. 2004. Development of heart valve leaflets and supporting apparatus in chicken and mouse embryos. Dev Dyn 230:239250. 10. 1002/dvdy. 20051

Liu R, Hu R, Zeng Y, Zhang W, and Zhou H-H. 2020. Tumour immune cell infiltration and survival after platinum-based chemotherapy in high-grade serous ovarian cancer subtypes: A gene expression-based computational study. EBioMedicine 51:102602-102602. 10.1016/j. ebiom. 2019. 102602

Lv Y, Zhao Y, Wang X, Chen N, Mao F, Teng Y, Wang T, Peng L, Zhang J, Cheng P, Liu Y, Kong H, Chen W, Hao C, Han B, Ma Q, Zou Q, Chen J, and Zhuang Y. 2019. Increased intratumoral mast cells foster immune suppression and gastric cancer progression through TNF-a-PD-L1 pathway. Journal for immunotherapy of cancer 7:54-54. 10. 1186/s40425-019-0530-3

Novruzov RM. 2008. Hemomicrocirculation channel and mastocyte population in outer oblique aponeurosis stomach muscles and uterus ligament in women with small pelvic organ prolapses. Georgian medical news:69-72.

Nygaard IE, Shaw JM, Bardsley T, and Egger MJ. 2014. Lifetime physical activity and pelvic organ prolapse in middle-aged women. Am J Obstet Gynecol 210:477. e471-412. 10. 1016/j. ajog. 2014. 01.035

Ottaiano A, Scala S, Normanno N, Botti G, Tatangelo F, Di Mauro A, Capozzi M, Facchini S, Tafuto S, and Nasti G. 2020. Prognostic and Predictive Role of CXC Chemokine Receptor 4 in Metastatic Colorectal Cancer Patients. Applied immunohistochemistry \& molecular morphology : AIMM: 10. 1097/PAI. 0000000000000828. 10.1097/PAI. 0000000000000828

Patadia M, Dixon J, Conley D, Chandra R, Peters A, Suh LA, Kato A, Carter R, Harris K, Grammer L, Kern R, and Schleimer R. 2010. Evaluation of the presence of $\mathrm{B}-$ cell attractant chemokines in chronic rhinosinusitis. American journal of rhinology \& allergy 24:11-16. 10. 2500/a jra. 2010. 24. 3386

Pegg AE. 2008. Spermidine/spermine-N(1)-acetyltransferase: a key metabolic regulator. American journal of physiology Endocrinology and metabolism 
456

457

458

459

460

461

462

463

464

465

466

467

468

469

470

471

472

473

474

475

476

477

478

479

480

481

482

483

484

485

486

487

488

489

490

491

492

493

494

294:E995-E1010. 10. 1152/ajpendo. 90217. 2008

Pegg AE. 2016. Functions of Polyamines in Mammals. J Biol Chem 291:14904-14912. 10. 1074 /jbc. R116. 731661

Qu S, Yang L, and Liu Z. 2020. MicroRNA-194 reduces inflammatory response and human dermal microvascular endothelial cells permeability through suppression of TGF- $\beta$ /SMAD pathway by inhibiting THBS1 in chronic idiopathic urticaria. J Cell Biochem 121:111-124. 10. 1002/jcb. 28941

Sajay-Asbaghi M, Sadeghi-Shabestrai M, Monfaredan A, Seyfizadeh N, Razavi A, and Kazemi T. 2020. Promoter region single nucleotide polymorphism of siglec-8 gene associates with susceptibility to allergic asthma. Personalized medicine:10.2217/pme-2018-0080. 10.2217/pme-2018-0080

Teicher BA, and Fricker SP. 2010. CXCL12 (SDF-1)/CXCR4 pathway in cancer. Clin Cancer Res 16:2927-2931. 10. 1158/1078-0432. CCR-09-2329

Türei D, Korcsmáros T, and Saez-Rodriguez J. 2016. OmniPath: guidelines and gateway for literature-curated signaling pathway resources. Nat Methods 13:966-967. 10. 1038/nmeth. 4077

Wang D, Wang X, Si M, Yang J, Sun S, Wu H, Cui S, Qu X, and Yu X. 2020a. Exosomeencapsulated miRNAs contribute to CXCL12/CXCR4-induced liver metastasis of colorectal cancer by enhancing M2 polarization of macrophages. Cancer letters 474:36-52. 10.1016/j. canlet. 2020.01.005

Wang H, Chen G, Wang H, and Liu C. 2013. RITA inhibits growth of human hepatocellular carcinoma through induction of apoptosis. Oncol Res 20:437445. 10. 3727/096504013x13685487925059

Wang M, Wang J, Liu J, Zhu L, Ma H, Zou J, Wu W, and Wang K. 2020b. Systematic prediction of key genes for ovarian cancer by co-expression network analysis. J Cell Mol Med. 10.1111/jcmm. 15271

Wang Y, He T, Herman JG, Linghu E, Yang Y, Fuks F, Zhou F, Song L, and Guo M. 2017. Methylation of ZNF331 is an independent prognostic marker of colorectal cancer and promotes colorectal cancer growth. Clin Epigenetics 9:115. 10. 1186/s13148-017-0417-4

Wu JM, Hundley AF, Fulton RG, and Myers ER. 2009. Forecasting the prevalence of pelvic floor disorders in U.S. Women: 2010 to 2050. Obstet Gynecol 114:1278-1283. 10.1097/AOG. 0b013e3181c2ce96

Yu J, Liang QY, Wang J, Cheng Y, Wang S, Poon TC, Go MY, Tao Q, Chang Z, and Sung JJ. 2013. Zinc-finger protein 331, a novel putative tumor suppressor, suppresses growth and invasiveness of gastric cancer. Oncogene 32:307317. 10. 1038/onc. 2012. 54

Yu Q, Vazquez R, Zabadi S, Watson RR, and Larson DF. 2010. T-1ymphocytes mediate left ventricular fibrillar collagen cross-linking and diastolic 
495

496

497

498

499

500

501

502

503

504

505

506

507

508

509

510

511

512

dysfunction in mice. Matrix Biol 29:511-518. 10.1016/j. matbio. 2010.06. 003

Zhang B, Wang Q, Fu C, Jiang C, and Ma S. 2019. Exploration of the immunerelated signature and immune infiltration analysis for breast ductal and lobular carcinoma. Annals of translational medicine 7:730-730. 10. 21037/atm. 2019. 11. 117

Zhao C, Isenberg JS, and Popel AS. 2018. Human expression patterns: qualitative and quantitative analysis of thrombospondin-1 under physiological and pathological conditions. J Cell Mol Med 22:2086-2097. 10.1111/jcmm. 13565 Zhou Z, Cheng Y, Jiang Y, Liu S, Zhang M, Liu J, and Zhao Q. 2018. Ten hub genes associated with progression and prognosis of pancreatic carcinoma identified by co-expression analysis. Int $J$ Biol Sci 14:124-136. 10. $7150 /$ i jbs. 22619

Zhu Q, Luo R, Gu J, Hou Y, Chen Z, Xu F, Wang L, Mao W, Lu C, and Ge D. 2020. High CXCR4 Expression Predicts a Poor Prognosis in Resected Lung Adenosquamous Carcinoma. Journal of Cancer 11:810-818. 10.7150/jca. 36498 
Figure 1

figure1

FIGURE 1. Workflow diagram. Data collection, analysis and validation.

Downloading data of GSE12852 from GEO database as training dataset

Remove outlier sample of GSE12852 dataset by "flash cluster" package of $\mathrm{R}$ software

Construct co-expression network and screen hub module

Construct PPI network using genes in hub module
GO and KEGG enrichment analysis for hub module

Screen hub genes and validate hub genes using the validation dataset

CIBERSORT algorithm characterized the proportion of 22 immune cells in samples from GSE12852 dataset

Investigation the differential immune cells infiltration between POP tissues and non-POP tissues 
Figure 2

figure2

FIGURE 2. Clustering dendrogram of 34 POP samples in GSE12852. POP samples were assigned as 1 ; non-POP samples were assigned as 0 . Pre-menopause status samples were assigned as 0; menopause status samples were assigned as 1 . Red color intensity is proportional to POP samples, pre-menopause status, and higher age.

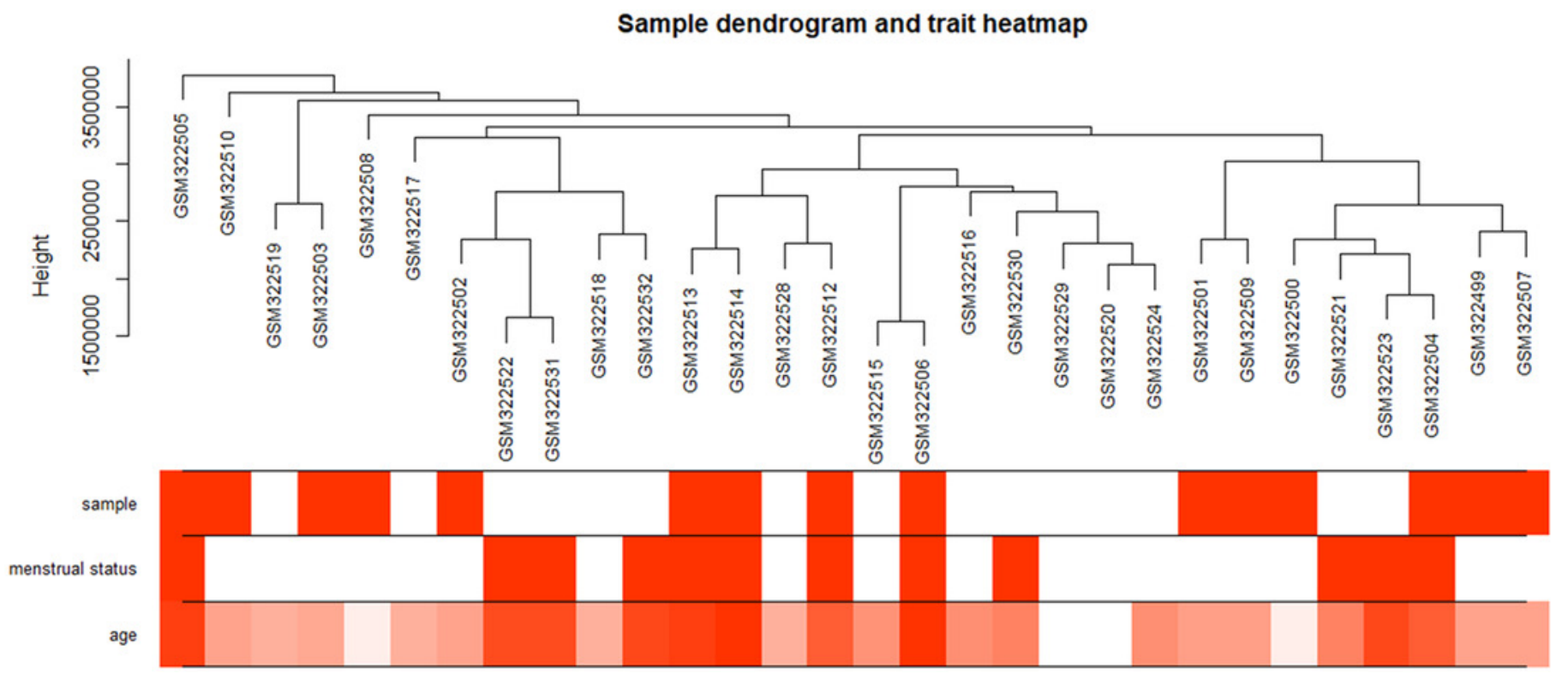




\section{Figure 3}

figure3

FIGURE 3. Identification of the soft threshold in the scale-free network. (A) Different soft-threshold and corresponding scale free topology model(B) Different soft-thresholding powers and corresponding mean connectivity. (C) The distribution of the connectedness by histogram. (D) Inspection of the scale free topology. 


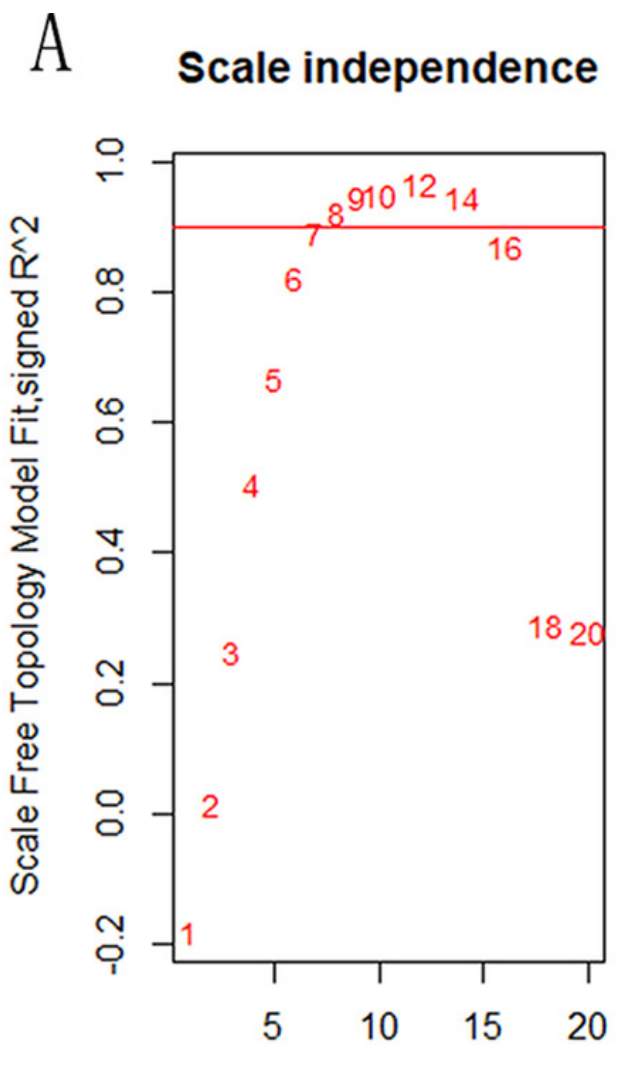

Soft Threshold (power)

C

Histogram of $\mathbf{k}$

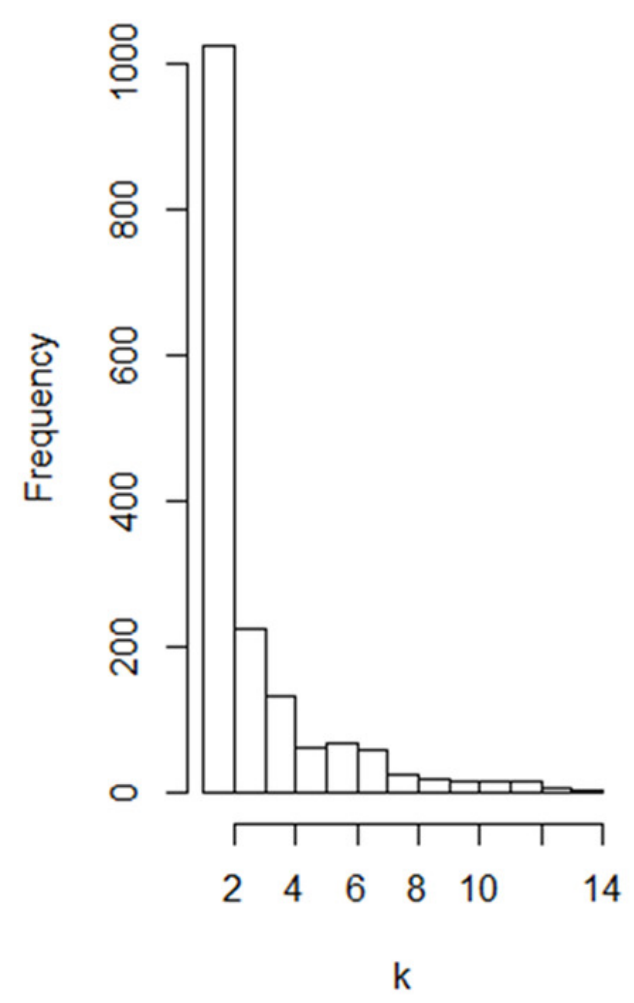

B Mean connectivity

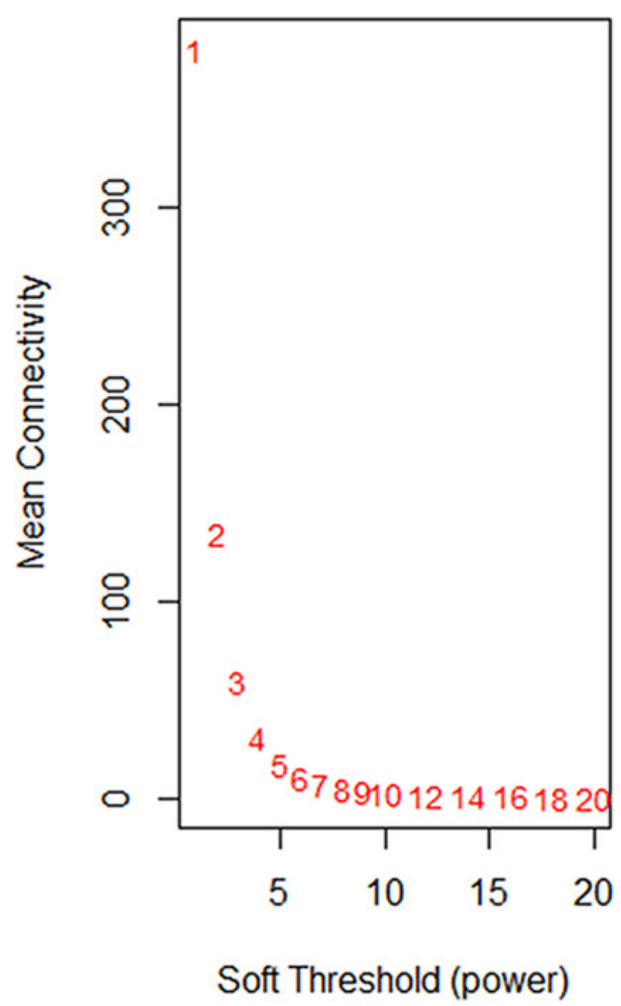

D check scale free topology scale $R^{\wedge} 2=0.98$, slope $=-\mathbf{2 . 0 3}$

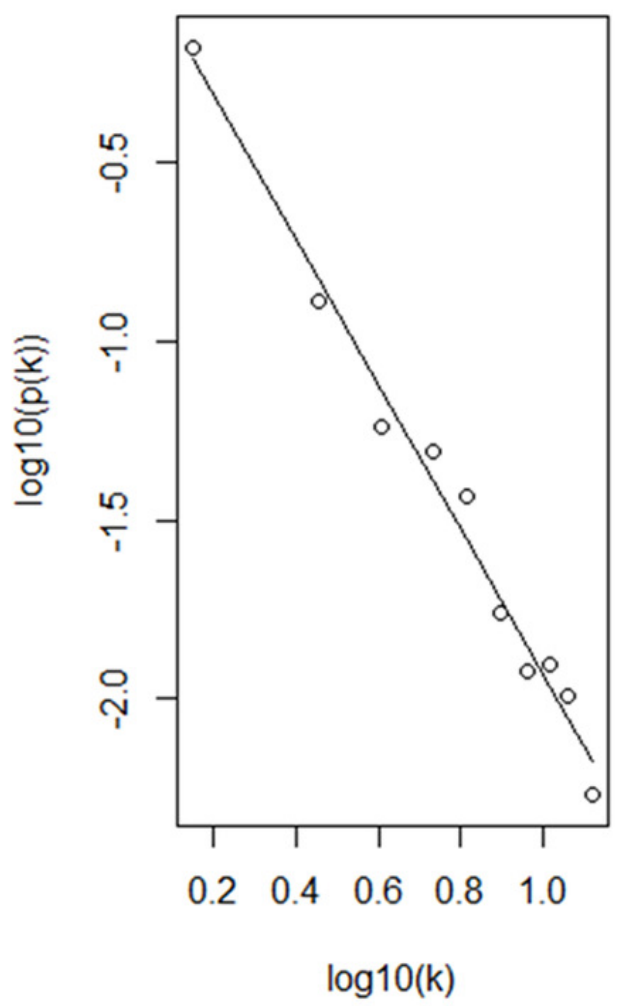


Figure 4

figure4

FIGURE 4. Identification of the hub module. (A) Dendrogram showed the genes with similar function classed into the same module according to the dissimilarity measure. (B) Heatmap revealed the correlation between modular eigengenes (ME) and clinical information of POP. 


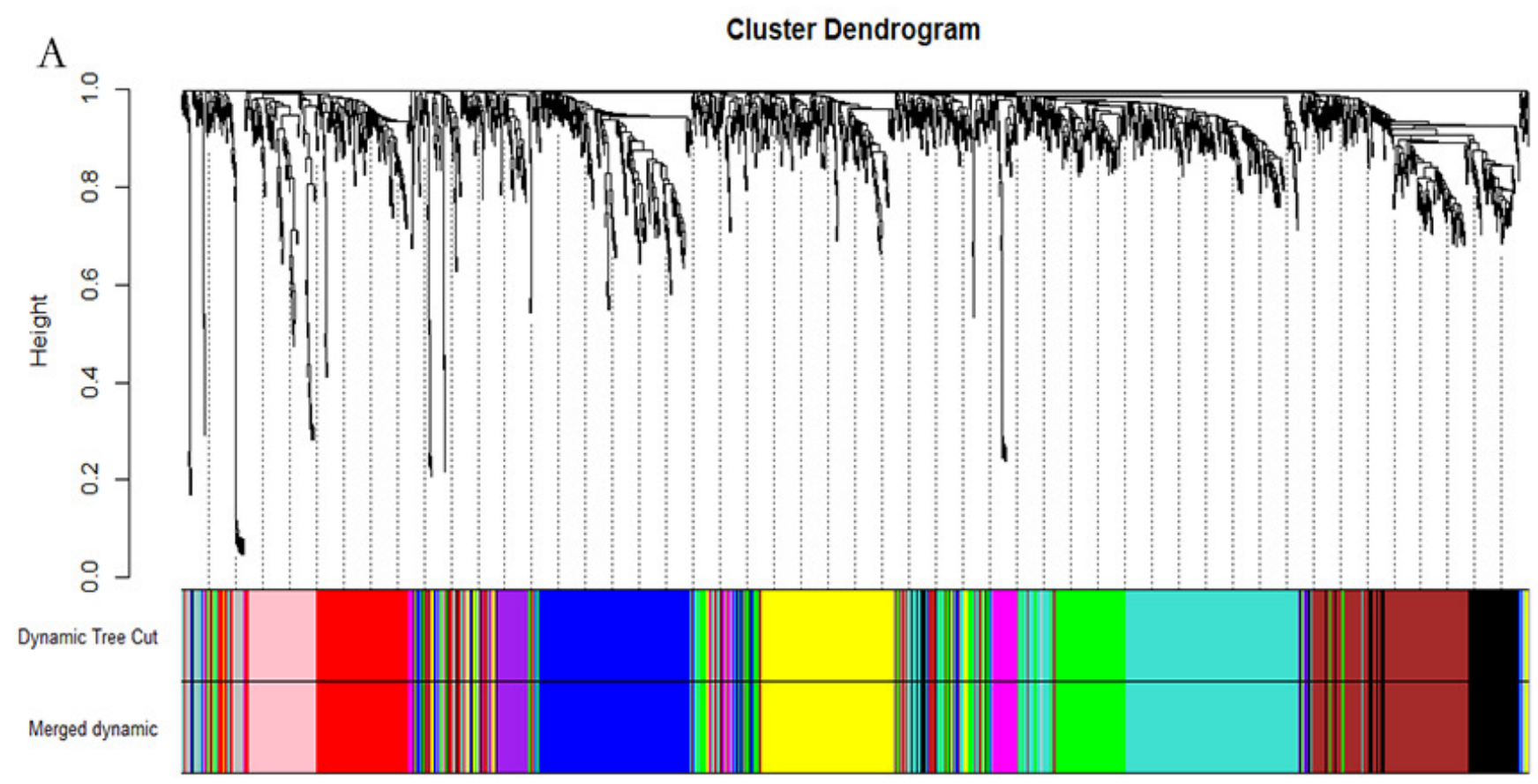

B

Module-trait relationships

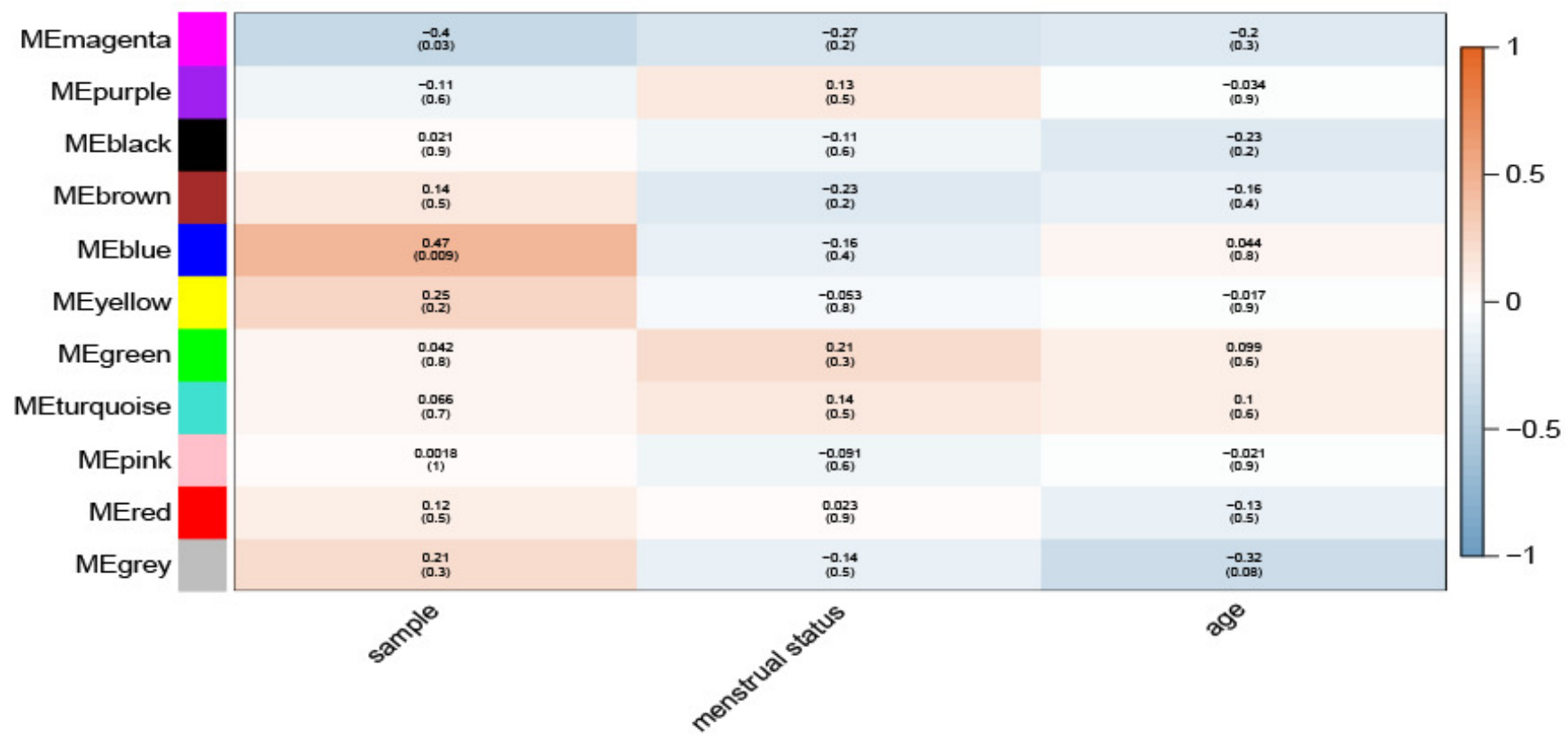




\section{Figure 5}

figure5

FIGURE 5. PPI network analysis. PPI network analysis of the genes in the blue module based on the OmniPath online database. Nodes represent the hub genes in the blue module identified by WGCNA. Lines represent interactions between hub genes. 


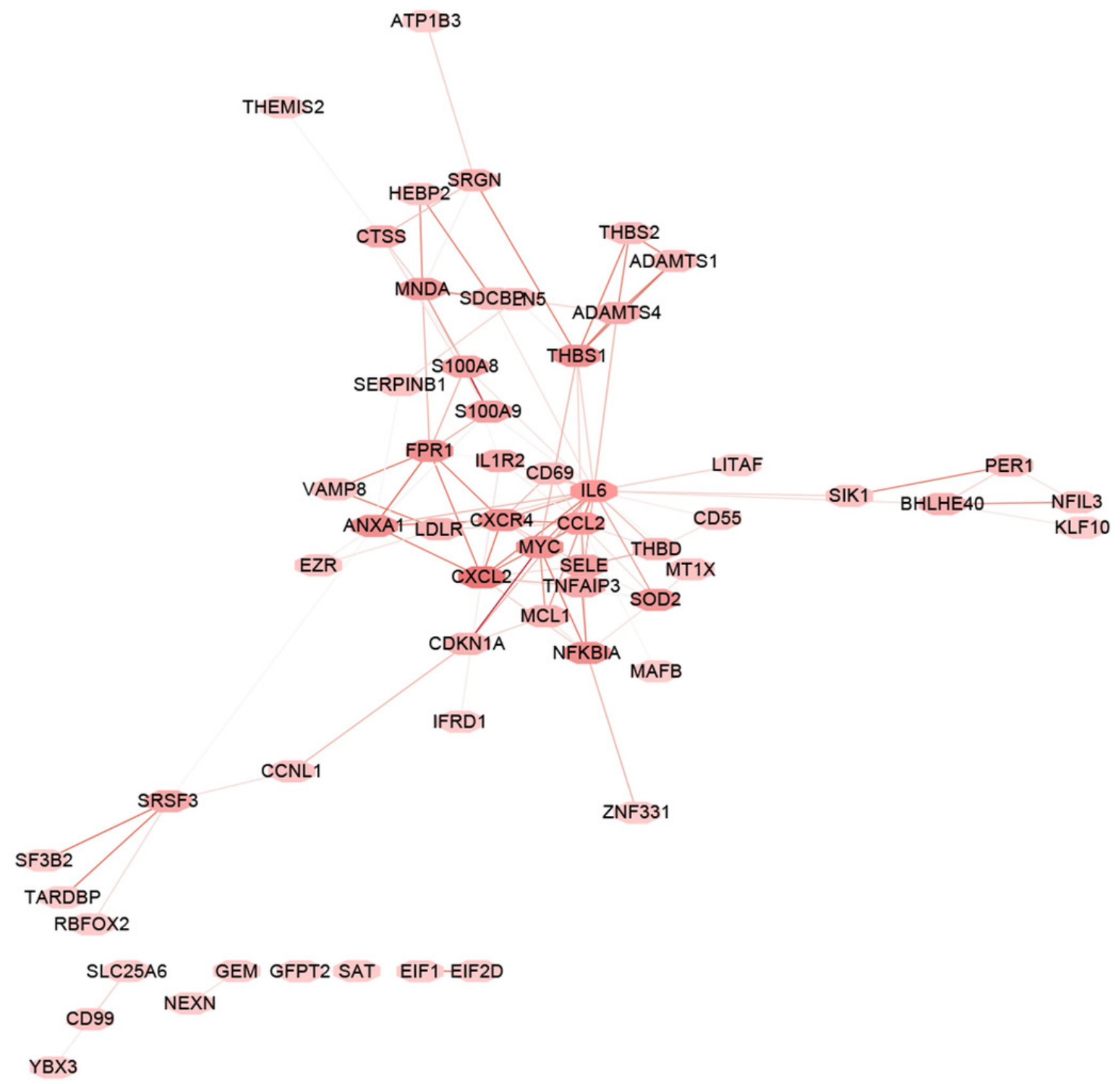


Figure 6

figure6

FIGURE 6. GO functional and KEGG pathway enrichment analysis. (A) Biological

process (BP), (B)cellular component (CC) and (C) molecular function (MF) of GO analyzed by the "org.Hs.eg.db" package in the R software. (D) KEGG pathway enrichment analysis using the "org.Hs.eg.db" package in the R software.
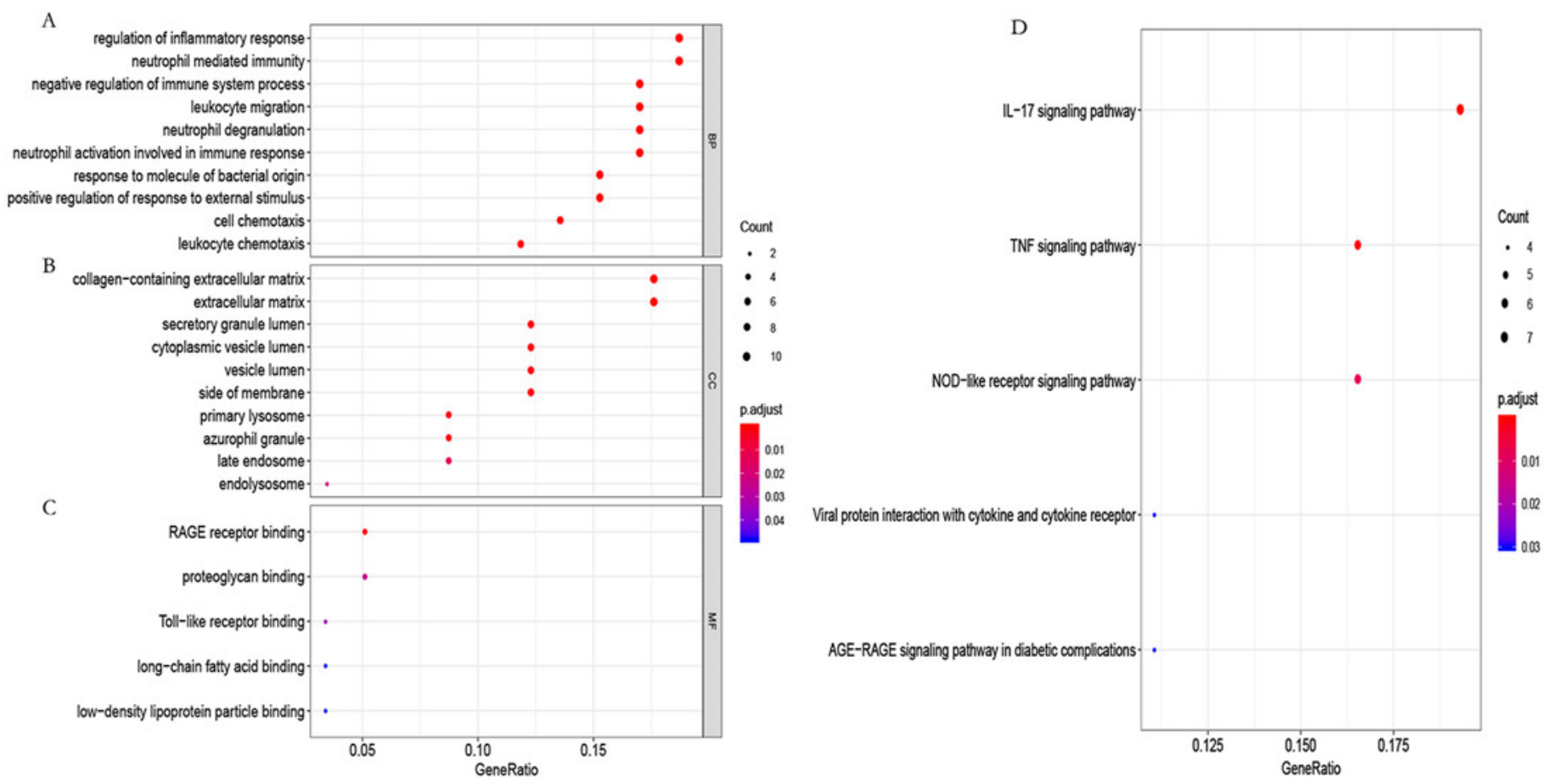
Figure 7

figure7

FIGURE 7. Selected hub gene. (A) The relationship between gene MM and GS. (B)

Differential expression of the hub genes. (C) Heatmap of the hub genes in GSE12852. (D) Correlation analysis of the hub genes in GSE12852.
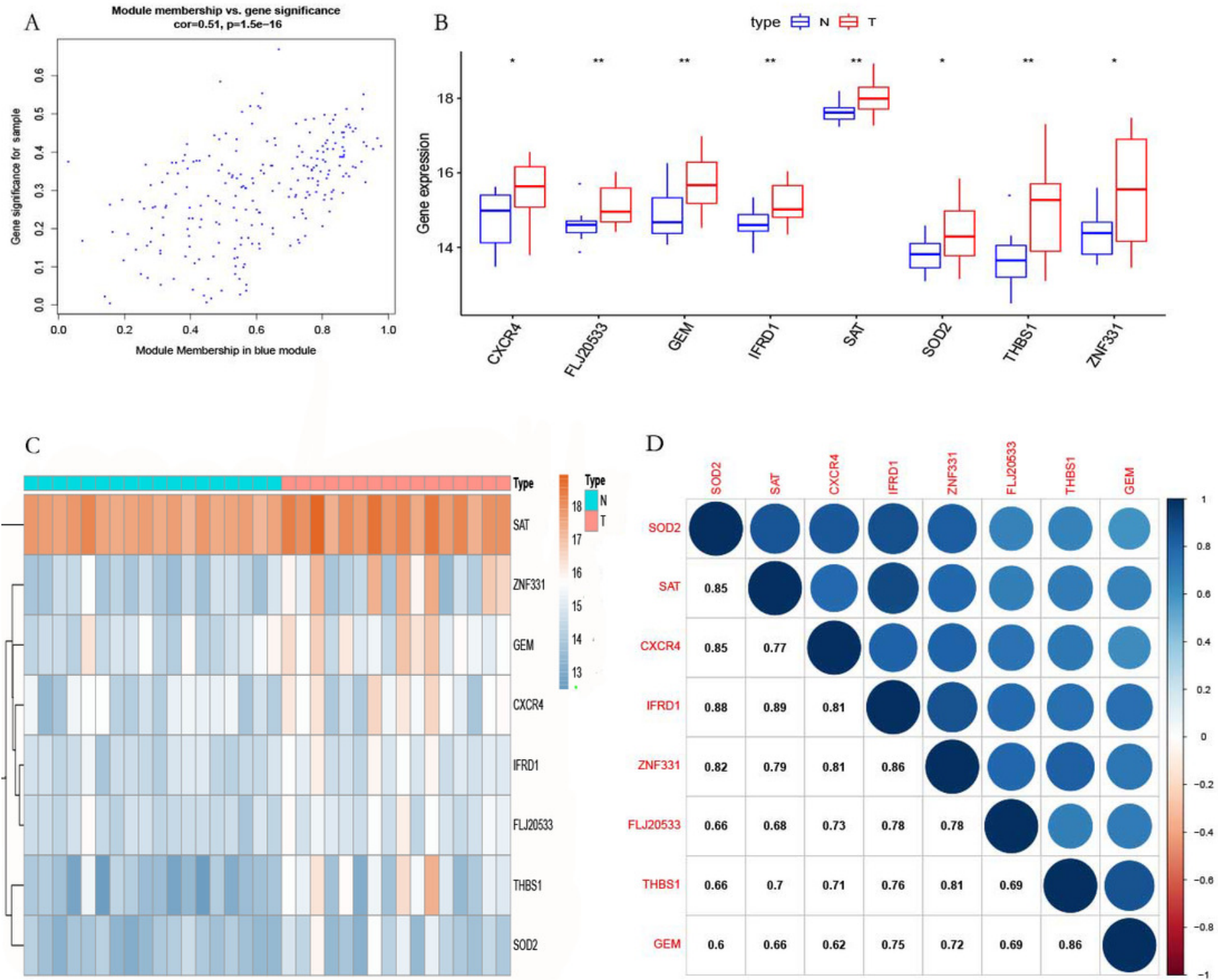
Figure 8

figure8

FIGURE 8. Hub gene validation. (A-H) RT-qPCR detection of the expression of hub genes in 12 pairs of POP and non-POP tissues.
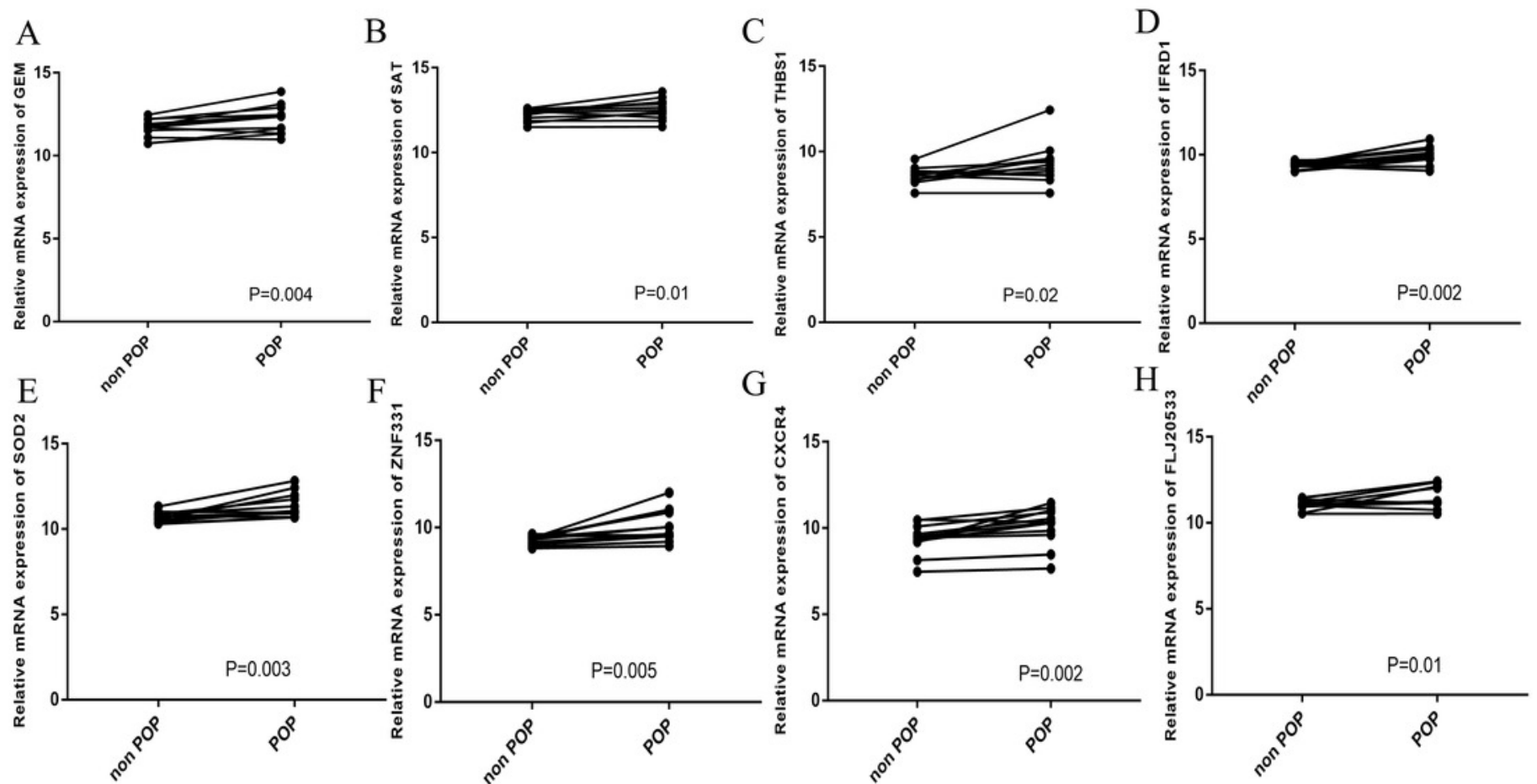
Figure 9

figure9

FIGURE 9. The landscape of immune cell infiltration in GSE12852 (CIBERSORT $p$ value < 0.05). (A) Proportion of the 22 immune cell types in GSE12852. (B) Correlation matrix between the 22 immune cell types. 


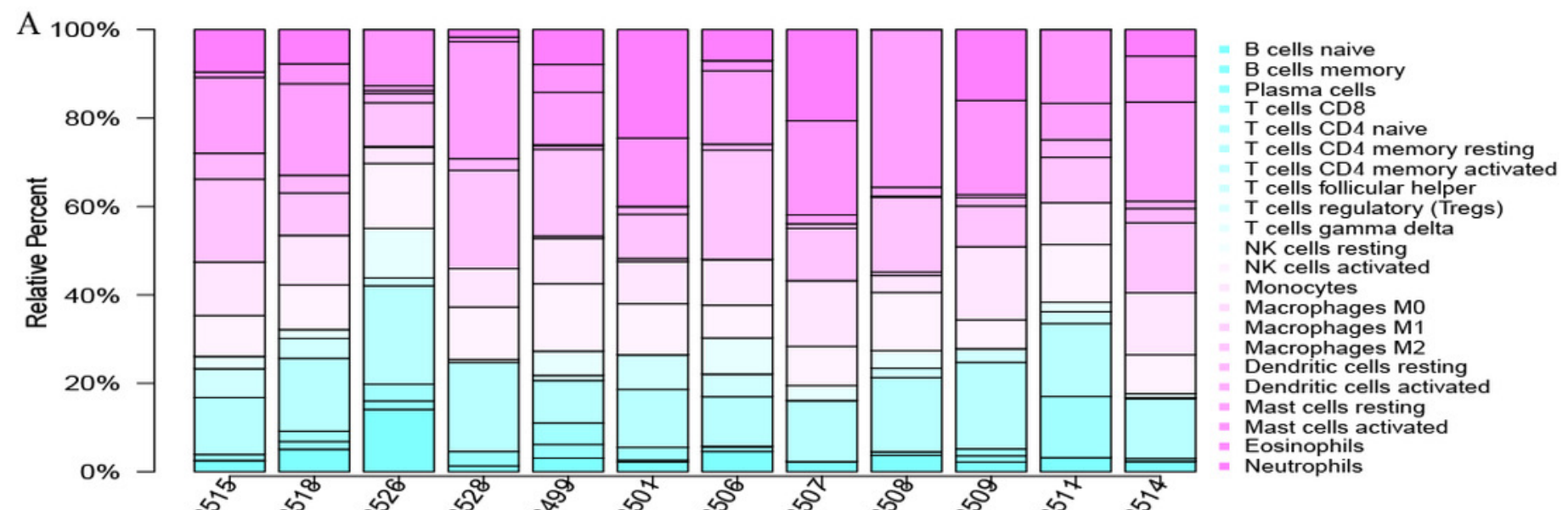

B

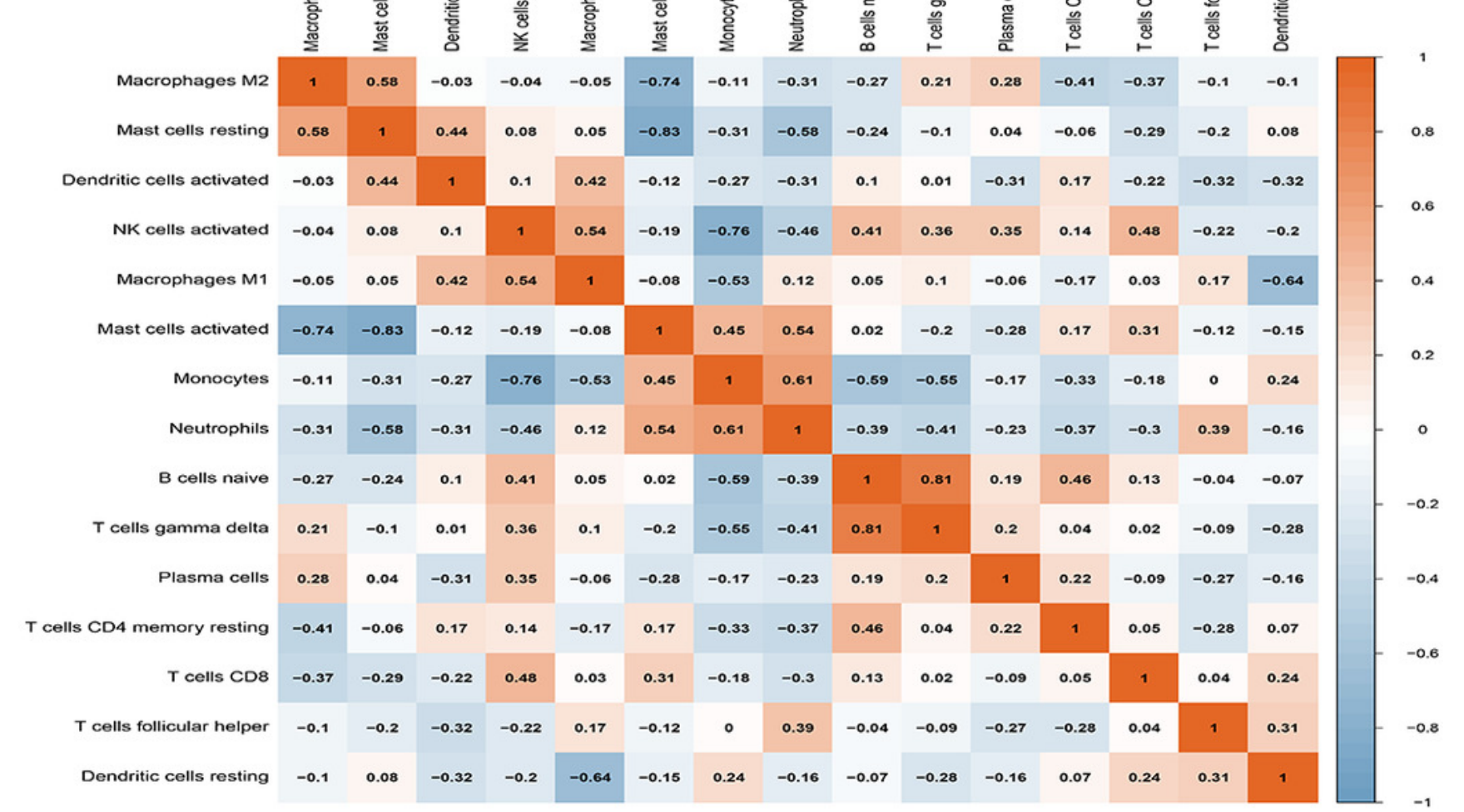




\section{Figure 10}

figure10

FIGURE 10. The differential immune cell infiltration between POP and non-POP

tissues. (A) Resting mast cells; (B) activated mast cells; (C) neutrophils. Group 1: non-POP; Group 2: POP.
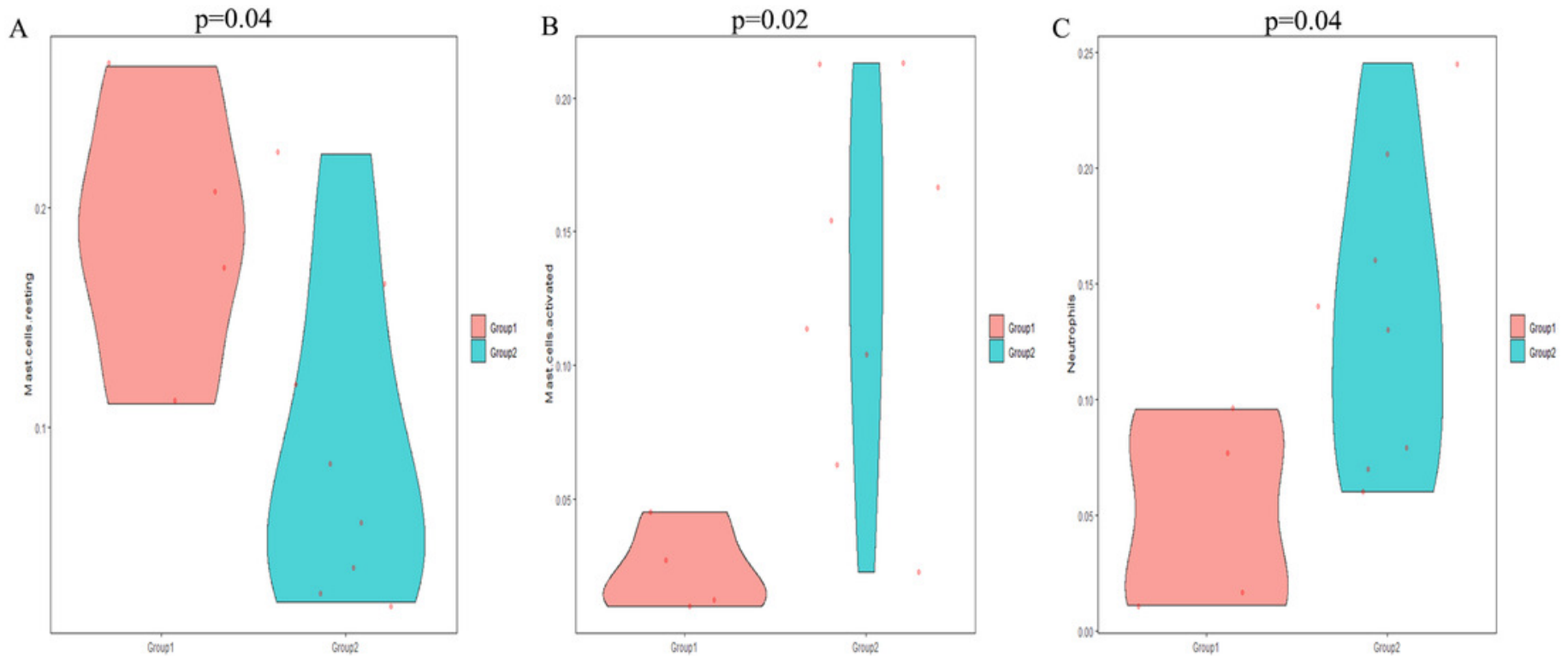


\section{Table $\mathbf{1}$ (on next page)}

table1

TABLE 1. The correlation between hub genes and immune cell infiltration 


\begin{tabular}{|l|l|r|r|}
\hline GENE & IIMMUNE CELL & P & R \\
\hline CXCR4 & Mast cells activated & 0.026025009 & 0.636596855 \\
\hline FLJ20533 & Mast cells activated & 0.037410947 & 0.604312579 \\
\hline GEM & Mast cells activated & 0.00382779 & 0.763874376 \\
\hline IFRD1 & Mast cells activated & 0.010102021 & 0.707220887 \\
\hline SAT & Mast cells activated & 0.026678483 & 0.634493564 \\
\hline SOD2 & Mast cells activated & 0.019559382 & 0.659816924 \\
\hline THBS1 & Mast cells activated & 0.023473376 & 0.645196019 \\
\hline ZNF331 & Mast cells activated & 0.005806179 & 0.741209161 \\
\hline GEM & Mast cells resting & 0.044390877 & -0.587895781 \\
\hline IFRD1 & Mast cells resting & 0.010475671 & -0.704821204 \\
\hline SAT & Mast cells resting & 0.029213165 & -0.626671202 \\
\hline SOD2 & Mast cells resting & 0.002740399 & -0.780472841 \\
\hline CXCR4 & Neutrophils & 0.002884121 & 0.778019124 \\
\hline FLJ20533 & Neutrophils & 0.009179588 & 0.713441726 \\
\hline GEM & Neutrophils & 0.030016479 & 0.624294555 \\
\hline IFRD1 & Neutrophils & 0.001688177 & 0.802313109 \\
\hline SAT & Neutrophils & 0.001387584 & 0.810472605 \\
\hline SOD2 & Neutrophils & $2.12 \mathrm{E}-04$ & 0.872716498 \\
\hline THBS1 & Neutrophils & 0.014406502 & 0.682777143 \\
\hline ZNF331 & Neutrophils & 0.002414909 & 0.786415881 \\
\hline
\end{tabular}

